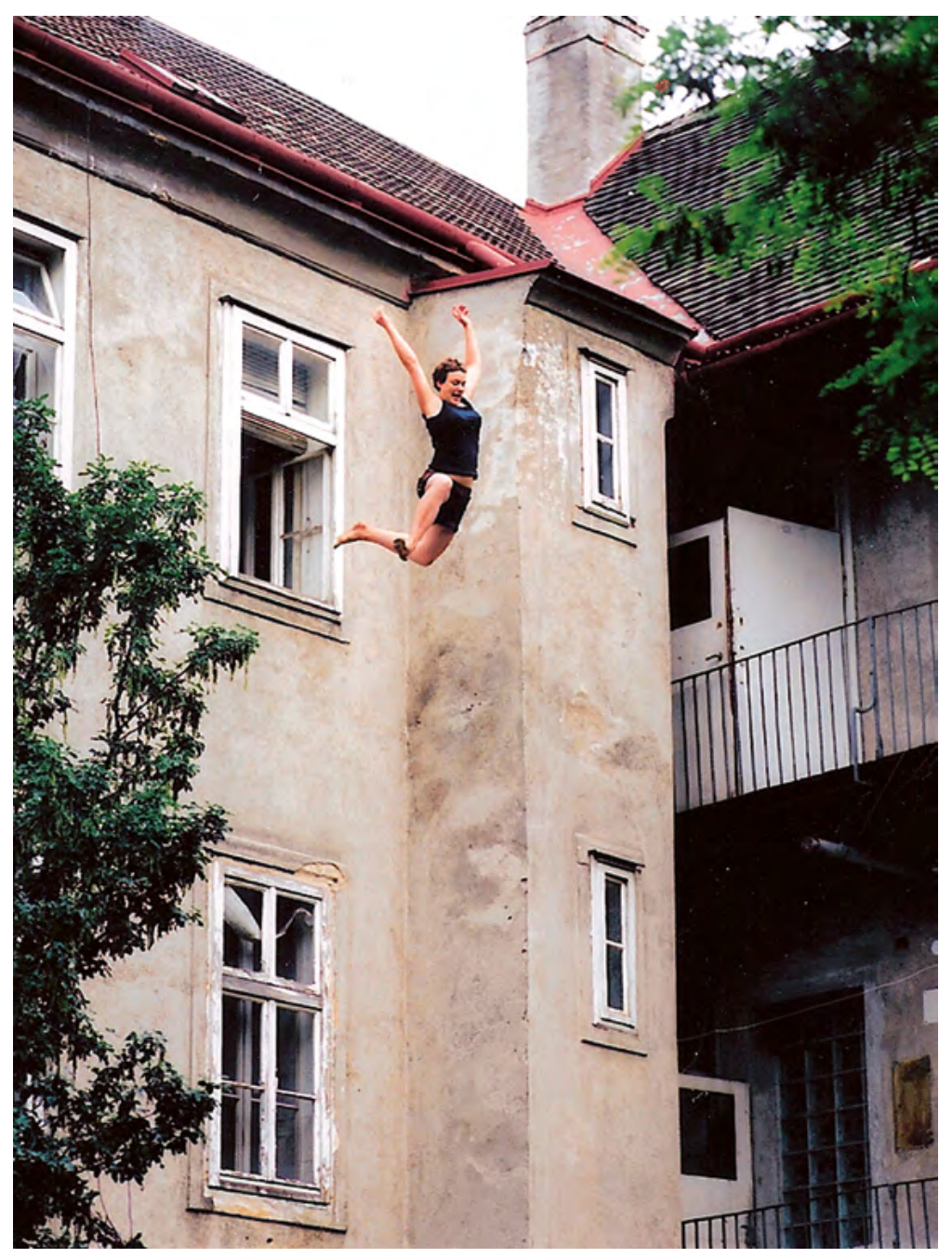

Référence bibliographique :

Corentin Fivet et Denis Zastavni, "Donner forme aux forces", lieuxdits\#1, juin 2011, pp.18-21. 


\section{Donner forme aux forces}

Un outil pour manipuler géométries et efforts

au sein des structures architecturales

Corentin Fivet
et Denis Zastavni

Postulons que la qualité des structures architecturales est intimement liée aux outils et méthodes employés par les concepteurs. Par conséquent, une des conditions pour qu'un système structural soit pertinent, efficace, robuste ou durable, consiste à utiliser des outils et méthodes intégrant ou favorisant l'accomplissement de ces objectifs.

\section{Conception structurale}

L'émergence des logiciels de modélisation et de calcul numérique il y a plusieurs dizaines d'années et les progrès encore effectués depuis ont abolis une grande part de la complexité mathématique inhérente à la conception des structures. Ainsi, les systèmes structurels en deviennent davantage contrôlés et les outils offrent une plus grande liberté formelle. Ainsi, ils se spécialisent pour servir distinctement (1) l'analyse, (2) l'optimisation, (3) le form-finding ou (4) la modélisation purement formelle.

Toutefois, la pertinence de l'utilisation de chacun de ces types d'outils pour la définition initiale des géométries de l'ouvrage peut être sujette à questionnement :

(1) Tout d'abord, les outils d'analyse. Ils dimensionnent ou vérifient les sections d'un système géométriquement prédéfini. On peut regretter le fait que ceuxci conduisent souvent le concepteur à figer les choix initiaux, ne facilitant pas la ré-interrogation et la ré-orientation des géométries ou du comportement structural.

(2) Les outils d'optimisation quant à eux agissent sur un modèle qui est déjà structurellement bien défini et n'y tra- vaillent qu'une sélection de paramètres pour fournir une solution unique dite optimale, vis-à-vis d'un critère mathématique tout autant particulier. Les modifications géométriques qui en découlent y sont donc des conséquences et non des choix premiers du concepteur, rendant difficile toute adaptation formelle ultérieure.

(3) Les outils de form-finding (itération de modifications géométriques d'une configuration initialement amorphe et non-équilibrée) sont assez similaires en ce sens où l'influence du concepteur sur la structure ne se fait qu'avant la mise en route du processus. L'ensemble des opérations intermédiaires étant enfermées dans une boîte noire, le résultat peut représenter une surprise qu'il s'agit souvent d'interpréter a posteriori.

(4) Hormis les modèles physiques et le travail au crayon, les autres outils fabriquant des formes ne prennent généralement pas en compte la réalité physique de la structure. Les solutions structurales nécessaires pour faire tenir de telles formes forcent un comportement structural non-adapté et utilisent systématiquement une quantité de matériau excessive et des détails coûteux.

\section{Morphogenèse structurale}

II apparaît donc primordial pour le concepteur de rester maître de toute la complexité du processus de conception, et ce dès les toutes premières définitions des géométries et des efforts internes c'est-à-dire avant toute analyse ou optimisation ultérieure, au moment de la morphogenèse structurale.

Cette approche n'est pas nouvelle. Par 


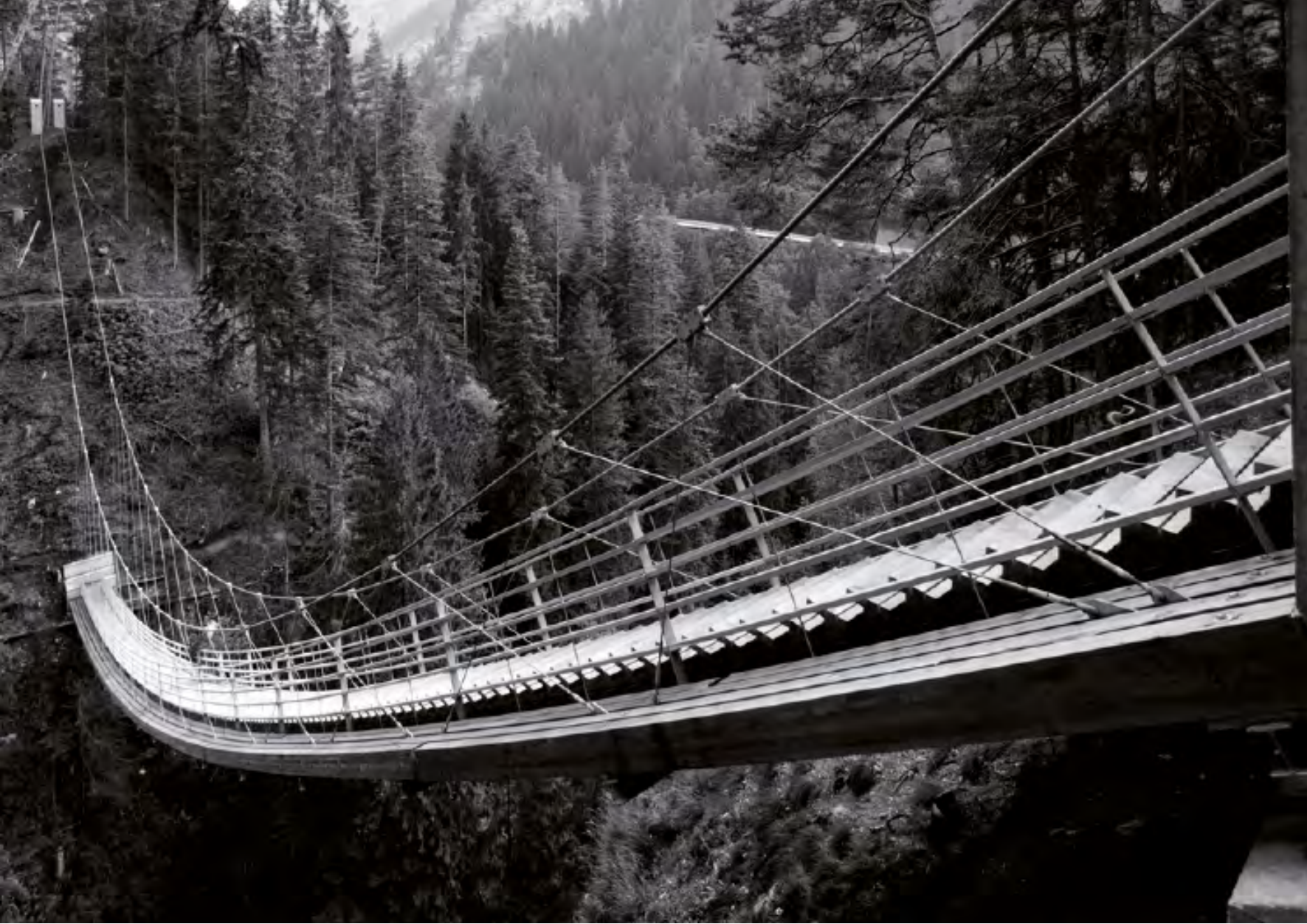

exemple, la méthode de travail de Robert Maillart visait systématiquement l'établissement préalable du principe structural dominant puis la mise en oeuvre des géométries pour le rendre effectif afin de viser une efficacité maximale dans l'emploi des matériaux. Félix Candela quant à lui faisait usage récurrent de géométries régulières pour simplifier les formulations mathématiques de ses coques. Plus populaires sont les maquettes physiques qui servaient Antonio Gaudi pour la définition des géométries de ses voûtes nervurées.

Des approches similaires peuvent être retrouvées chez d'autres concepteurs de l'ère préinformatique comme Eugène Freyssinet, John Roebling, Eladio Dieste, Pier Lugi Nervi ou Otto Frei mais également chez des concepteurs contemporains comme Jürg Conzett, Laurent Ney, Aurelio Muttoni ou Jörg Schlaich.

\section{Morphogenèse structurale assistée par ordinateur}

La recherche présentée ici s'inscrit dans ce contexte. Initiée en septembre 2009 par Corentin Fivet sous la direction de Denis Zastavni et de Jean-François Cap, elle a pour objet la constitution de méthodes graphiques destinées à accompagner le concepteur lors de la première définition du système structural. L'approche suivie privilégie : (1) la manipulation graphique et simultanée des géométries de l'ouvrage et de leurs incidences mécaniques ; (2) l'emploi de simplifications théoriques permettant la maîtrise globale du problème structural ; et (3) le contrôle initial et permanent du concepteur sur la structure en cours de définition.

L'usage de méthodes et d'outils simplificateurs est essentiel pour le concepteur, entre autres, pour éviter que sa créativité et sa supervision globale ne soient freinées par des calculs procéduraux trop longs. Cette décomplexification du problème structural est ici entreprise à l'aide de méthodes de statique graphique, du théorème conservateur $d u$ calcul plastique et de l'informatisation du processus.

La statique graphique est une méthode ayant la particularité d'être entièrement visuelle. Elle permet de manipuler simultanément les géométries de l'ouvrage, sur un premier diagramme, et l'intensité des efforts internes au sein de cet ouvrage, sur un second diagramme. Chaque barre de la structure est représentée par deux lignes, une dans chaque diagramme, toujours parallèles entre elles. Utiliser la statique graphique par le biais informatique augmente la précision du calcul (autrement dépendante de l'épaisseur de la mine de crayon) et la souplesse de manipulation de ses diagrammes. L'informatisation rend également possible la systématisation paramétrée de certaines procédures graphiques.

D'autre part, le théorème conservateur du calcul plastique nous apprend que s'il est possible, au sein d'un ouvrage, d'assurer le fonctionnement d'un mécanisme structural suffisant à reprendre les charges en présence, alors la stabilité de l'ouvrage est assurée quel que soit le comportement structural réellement adopté. Son interprétation élargit grandement le champs d'application de la statique graphique. Ce champ regroupe illustration

Pont du Traversina, par Conzett,

Bronzini, Gartmann AG

photo de Wilfried Dechan 
alors les structures réticulées métalliques ou en bois (isostatiques comme hyperstatiques), le travail des moments internes, les maçonneries, les éléments en béton armé, les structures en tenségrité, etc.

\section{Manipulation d'équilibre structural assistée par ordinateur}

Concrètement, l'outil proposé permet au concepteur d'effectuer, dans un environnement entièrement graphique, des suites d'opérations simples sur des systèmes structurels restant équilibrés à chaque étape. Chaque diagramme n'est composé que de barres, de forces et de contraintes géométriques (lignes, secteurs ou rayons maximaux), toutes manipulables en déplaçant à la souris un nombre restreint de noeuds.

Au départ, seules sont connues - et donc dessinées - les charges à appliquer, les éventuelles frontières géométriques - limitant le déploiement spatial de la structure - et les contraintes mécaniques - prenant en compte les propriétés de résistances maximales du matériau. Par étapes successives, il appartient alors au concepteur de donner forme à la structure à l'aide d'une dizaine d'opérations simples comme, par exemple : déplacer un noeud pour modifier l'orientation, l'intensité ou le point d'application d'un certain effort ; décomposer une force en deux composantes ; transformer deux forces opposées, alignées et de même intensité en une barre comprimée ou tendue ; dé- composer cette barre ou une autre en deux efforts opposés, etc.

Ces opérations peuvent être motivées par des raisons d'ordre formel (par visualisation du premier diagramme) ou d'ordre mécanique (par interprétation du second diagramme), mais leur manipulation se fera toujours par le déplacement géométrique de noeuds dont les positions au sein de leur diagramme respectif sont limitées par des domaines (sur un point, le long d'une ligne, au sein d'un secteur ou d'une surface) afin de satisfaire les contraintes géométriques et mécaniques initialement posées. Étant donné que ces domaines sont construits automatiquement par des constructions purement géométriques ne traitant que d'intersection, de copie ou de parallélisme, l'utilisateur reste visuellement attentif à la logique du fonctionnement interne des automatisations - la boîte noire n'existe pas.

Pratiquer un tel outil, c'est guider de manière intuitive le travail préliminaire de la définition des géométries de l'ouvrage structural en projet, simultanément à la définition des contraintes attendues au sein de cet ouvrage. En d'autres mots, s'il est question ici de donner forme aux forces... il s'agit également de donner force aux formes. 
recherche

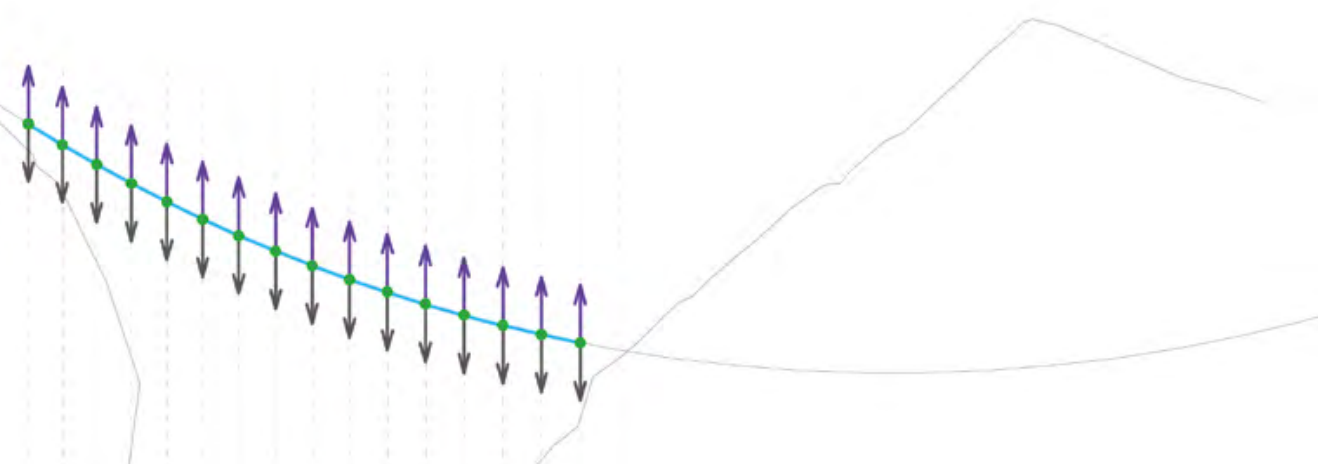

Avant toute opération géométrique.

Le tablier est équilibrépar des efforts temporaires (en mauve) devant être manipulésparl'utilisateur

Diagramme des géométries au-dessus et diagramme des efforts en-dessous. Dessin de l'auteur:

Après la dernière manipulation équilibrée.

Lesnoeuds vertspeuvent encore être déplacés (certains librement et d'autresle long des arcs de cercles). Les noeuds rouges et les géométries des barres sont automatiquement positionnés afin de garanti l'équilibre permanent de la structure. Dessin de l'auteur.

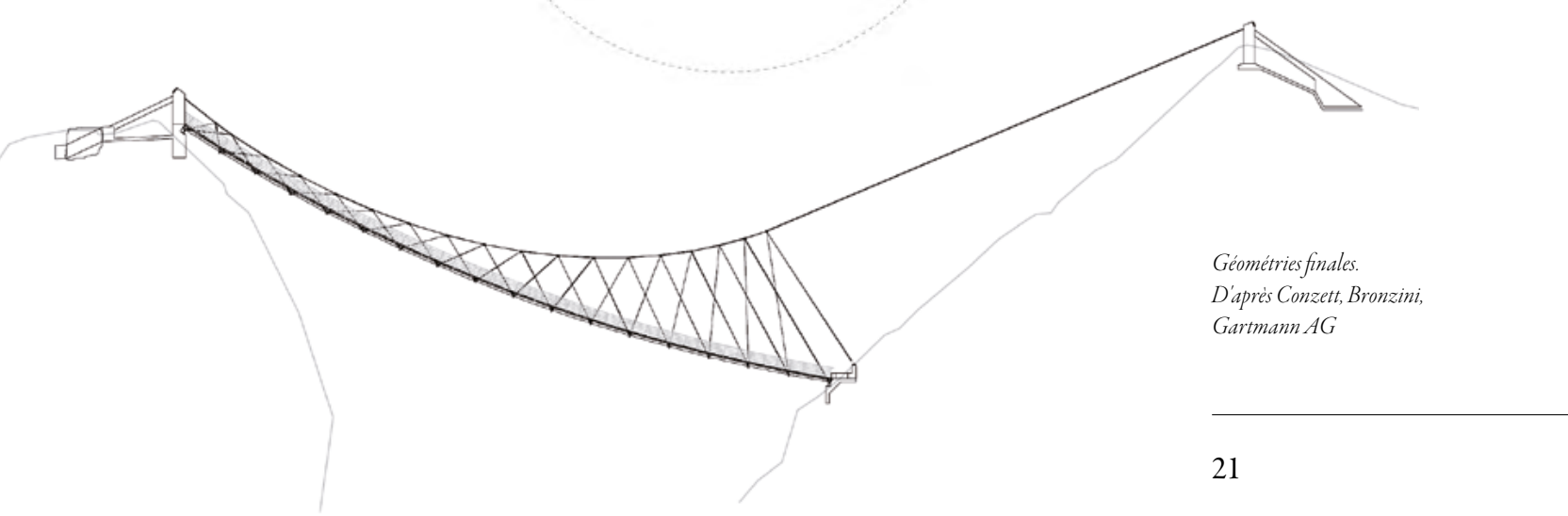

